\title{
ADAPTIVE OPTICS PHOTOMETRY AND ASTROMETRY OF BINARY STARS. II. A MULTIPLICITY SURVEY OF B STARS ${ }^{1}$
}

\author{
LEWIS C. ROBERTS, JR. \\ The Boeing Company, Kihei, HI, USA; lewis.c.roberts@boeing.com \\ AND \\ Nils H. Turner and Theo A. ten BrummelaAr \\ Center for High Angular Resolution Astronomy, Georgia State University, Mount Wilson, CA, USA; \\ nils@chara-array.org, theo@chara-array.org \\ Received 2006 October 3; accepted 2006 October 16
}

\begin{abstract}
We carried out a search for companions to B stars using the adaptive optics system of the Advanced ElectroOptical System $3.6 \mathrm{~m}$ telescope. We observed $70 \mathrm{~B}$ stars in the $I$ band, finding 16 companions, 10 of which are newly discovered companions. For each system with a detected companion we give its measured astrometry and differential magnitude. We then put the observation in context of the system's known multiplicity and provide a somewhat crude estimate of its spectral type based on the single differential magnitude measurement. The separations range from $0.2^{\prime \prime}$ to $7.31^{\prime \prime}$, and the differential magnitudes range from $1.41 \pm 0.01$ to $10.1 \pm 0.5$. Finally, we present a list of the null detections and discuss the implications of null detections of selected stars.
\end{abstract}

Key words: astrometry — binaries: close — binaries: visual — instrumentation: adaptive optics — techniques: photometric

\section{INTRODUCTION}

B stars form in clusters and undergo interactions with other cluster members. This often leads to disruption of binaries and the creation of new binaries. Knowing the differences in companion fraction between cluster members, field stars, and runaway stars allows us to better understand the collisional history of these stars. Also, a more complete understanding of the types of B star companions allows the determination of the companion initial mass function, which can differentiate between binary formation models.

There have been several surveys of B star duplicity in the past. Wolff (1978) carried out a spectroscopic survey of 83 late B-type stars and found that $24 \%$ of the stars had companions with mass ratios greater than 0.1 and orbital periods greater than 100 days. Additional companions showed some low-amplitude variations that might be indicative of low-mass companions. In a sample of 109 stars (all B2-B5 IV or V stars) Abt et al. (1990) detected 32 binaries spectroscopically and 49 visually for a binary frequency of $74 \%$. McAlister et al. $(1987,1993)$ carried out a speckle interferometry survey of the Bright Star Catalog and resolved 34 of 245 B IV and B V stars into binaries; this is a $13.9 \%$ multiplicity fraction. Later, Mason et al. (1997) conducted a speckle interferometry survey of Be stars covering 48 stars, showing that Be and B star binary fractions were similar, at $10 \% \pm 4 \%$ of the sample.

While these surveys have started to determine the binary frequency of B stars, they all have selection effects and have not fully surveyed the complete phase space of companions. Multiplicity surveys have used two main techniques, spectroscopic studies and speckle interferometry.

Spectroscopic surveys are biased toward finding close companions or ones that are a significant fraction of the primary's mass. Speckle interferometry is well suited for finding compan-

\footnotetext{
${ }^{1}$ Based on observations made at the Maui Space Surveillance System, operated by Detachment 15 of the US Air Force Research Laboratory's Directed Energy Directorate.
}

ions that have larger separations, but it has a limited dynamic range and cannot find companions that are much fainter than the primary. For main-sequence primaries, this translates into not being able to find late-type stars.

Adaptive optics (AO) is a technique that can help fill in this gap in coverage. It can detect much higher dynamic range companions than speckle interferometry at similar separation ranges. The instrumentation and techniques used in two of the more prolific speckle programs (Center for High Angular Resolution Astronomy [CHARA] and the US Naval Observatory) can only detect binaries with a maximum differential magnitude of 3.5 (Mason 1994), which for a B0 V is a B9 V companion and for a B8 V star is a G0 V companion (Cox 2000). With AO on the Advanced Electro-Optical System (AEOS) telescope we can detect companions $\sim 10$ mag fainter than the primary (N. Turner et al. 2007, in preparation). For main-sequence B stars this enables us to detect M-dwarf companions.

While no one technique can find all types of companions, in combination they can gather a complete understanding of the binary nature of B stars. This paper seeks to further increase our knowledge of the multiplicity of B stars by beginning a survey of B stars with AO.

\section{OBSERVATIONS}

Observations were made using the AEOS $3.6 \mathrm{~m}$ telescope and its AO system. The AEOS telescope is located at the Maui Space Surveillance System at the summit of Haleakala (Bradley et al. 2006). There were dedicated observing runs in 2001 February, 2001 September, 2002 March, and 2002 September. Stars were also observed on a queue-scheduled basis between 2001 and 2004. The observing run was focused on a multiplicity survey of $\mathrm{O}$ stars (N. Turner et al. 2007, in preparation). The B stars formed a secondary target list. Since the B stars were a secondary target, and there are so many more $\mathrm{B}$ stars than $\mathrm{O}$ stars, a much smaller percentage of the $\mathrm{B}$ stars were observed. 
TABLE 1

B StaR Binaries

\begin{tabular}{|c|c|c|c|c|c|c|c|}
\hline $\mathrm{HD}$ & WDS & Discovery & Hipparcos & Besselian & $\begin{array}{c}\rho \\
(\operatorname{arcsec})^{\mathrm{a}}\end{array}$ & $\begin{array}{c}\theta \\
(\mathrm{deg})^{\mathrm{b}}\end{array}$ & $\Delta I$ \\
\hline 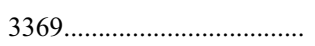 & $00369+3343$ & RBR 3 Aab & 2912 & 2001.7424 & 0.20 & $265.1 \mathrm{Z} / 97.6 \mathrm{~N}$ & $8.7 \pm 1.8^{\mathrm{c}}$ \\
\hline 5394 & $00567+6043$ & BU $1028 \mathrm{AB}$ & 4427 & 2002.6845 & 2.07 & 259.0 & $6.85 \pm 0.18$ \\
\hline $23862 \ldots \ldots \ldots \ldots \ldots \ldots$ & $03492+2408$ & RBR 4 Aab & 17851 & 2002.0924 & 4.66 & $221 \mathrm{Z} / 119 \mathrm{~N}$ & $9.4 \pm 0.5^{\mathrm{c}}$ \\
\hline $37711 \ldots \ldots \ldots$ & $05413+1632$ & BU 1007 & 26777 & 2001.0985 & 0.26 & 221.2 & $1.51 \pm 0.03^{\mathrm{d}}$ \\
\hline $44458 \ldots$ & $06214-1146$ & STF $3116 \mathrm{AB}$ & 30214 & 2003.9873 & 3.90 & 22.7 & $3.69 \pm 0.01$ \\
\hline $100600 \ldots \ldots \ldots \ldots \ldots \ldots$ & $11347+1648$ & STF $1552 \mathrm{AB}$ & 56473 & 2002.2407 & 3.41 & 205.8 & $1.41 \pm 0.01$ \\
\hline $106625 \ldots \ldots \ldots$ & $12158-1733$ & RBR $5 \mathrm{AB}$ & 59803 & 2002.2408 & 1.06 & 96.8 & $7.1 \pm 1.0^{\mathrm{c}}$ \\
\hline $120198 \ldots \ldots \ldots \ldots \ldots \ldots \ldots$ & $13466+5426$ & RBR 6 Aa & 67231 & 2002.2410 & 2.42 & 115.9 & $10.1 \pm 1.0^{\mathrm{c}}$ \\
\hline . & $15170-0923$ & $\mathrm{RBR} 7 \mathrm{AB}$ & 74785 & 2002.2411 & 2.09 & 194.2 & $8.8 \pm 0.5^{\mathrm{c}}$ \\
\hline 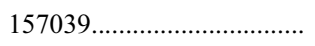 & $17227-3748$ & $\mathrm{~B} 908 \mathrm{AB}$ & 85020 & 2001.4686 & 2.71 & 114.5 & $6.0 \pm 0.1^{\mathrm{e}}$ \\
\hline \multirow[t]{2}{*}{$164863 \ldots \ldots \ldots \ldots \ldots \ldots \ldots \ldots \ldots \ldots \ldots \ldots \ldots$} & $18042-2230$ & B1975 BC & $\ldots$ & 2001.6710 & 0.47 & 236.0 & $4.90 \pm 0.30^{\mathrm{e}}$ \\
\hline & & & & 2002.6729 & 0.47 & 235.5 & $4.65 \pm 0.22$ \\
\hline $164906 \ldots \ldots \ldots$ & $18044-2423$ & RBR $8 \mathrm{AB}$ & & 2001.6710 & 3.14 & 318.3 & $8.04 \pm 0.37^{\mathrm{c}}$ \\
\hline $170938 \ldots \ldots \ldots$ & $18326-1542$ & $\mathrm{RBR} 9 \mathrm{AB}$ & 90907 & 2001.7364 & 7.31 & $250.2 \mathrm{Z} / 218.1 \mathrm{~N}$ & $7.23 \pm 0.22^{\mathrm{c}}$ \\
\hline $171432 \ldots \ldots \ldots \ldots \ldots \ldots \ldots \ldots \ldots \ldots$ & $18356-1833$ & RBR $10 \mathrm{AB}$ & 91143 & 2002.5474 & 4.86 & 320.0 & $10.6 \pm 0.5^{\mathrm{c}}$ \\
\hline $174638 \ldots \ldots \ldots$ & $18501+3322$ & RBR $11 \mathrm{Aa}$ & 92420 & 2002.6731 & 0.54 & 176.3 & $4.53 \pm 0.20^{\mathrm{c}}$ \\
\hline 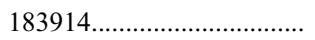 & $19307+2758$ & RBR $12 \mathrm{Ba}$ & 95951 & 2002.6842 & 0.39 & 107.5 & $4.12 \pm 0.17^{\mathrm{c}}$ \\
\hline
\end{tabular}

a Errors in this column are $\pm 0.02^{\prime \prime}$ for $\rho \leq 1^{\prime \prime}, \pm 0.01^{\prime \prime}$ for $1^{\prime \prime}<\rho \leq 4^{\prime \prime}$, and $\pm 0.02^{\prime \prime}$ for $\rho>4^{\prime \prime}$.

b The position angles all have an error of $\pm 2^{\circ}$.

${ }^{c}$ New discovery.

${ }^{d}$ This measurement was made using the $700-1060 \mathrm{~nm}$ filter.

e Confirmation observation.

An observing list of observable B stars was compiled from the SIMBAD database. ${ }^{2}$ Stars were chosen so that they had zenith angles of $\leq 45^{\circ}$ (declinations from $-24.3^{\circ}$ to $+65.75^{\circ}$ ) and were brighter than 9 mag in the $V$ band. The brightness limit was chosen because the AEOS AO system has a limiting magnitude of approximately 9 mag, although the exact limit depends on atmospheric conditions. This created a list with 3113 stars. In the end we observed 70 stars, or $\sim 2.2 \%$ of the available stars. The small percentage of the available targets that were observed limits the amount of statistical analysis that can be applied the sample.

The AEOS AO system is a natural guide star system using a Shack-Hartmann wave front sensor (Roberts \& Neyman 2002). The individual subaperatures have a diameter of $11.9 \mathrm{~cm}$ projected onto the primary. The deformable mirror has 941 actuators. The system's closed-loop bandwidth is adjustable and can run up to $200 \mathrm{~Hz}$, although the normal range is approximately $50 \mathrm{~Hz}$. In the configuration used for these observations, the light from 500 to $540 \mathrm{~nm}$ is sent to the tip-tilt detector system, the light from 540 to $700 \mathrm{~nm}$ is sent to the wave front sensor, and the light longer than $700 \mathrm{~nm}$ is sent to the Visible Imager CCD science camera.

Each data set consists of 1000 frames using a Bessel $I$-band filter. After collection, any saturated frames are discarded, and the remaining frames are debiased, dark-subtracted, and flat-fielded. The frames are weighted by their peak pixel, which is proportional to their Strehl ratio, and then co-added using a shift-and-add routine. The resulting image is analyzed with the program fitstars; it uses an iterative blind deconvolution that fits the location of delta functions and their relative intensity to the data. The co-adding technique and the analysis with fitstars were first presented in ten Brummelaar et al. (1996). Upgrades to fitstars were discussed in ten Brummelaar et al. (2000) and Roberts et al. (2005).

Error bars on the astrometry and photometry were assigned using the method in Roberts et al. (2005). For the photometry, simulated binary stars were created from observations of single stars. The photometry of these simulated binaries was measured

\footnotetext{
${ }^{2}$ The SIMBAD database can be found at http://simbad.u-strasbg.fr.
}

and used to create a grid of measurement errors as a function of separation and differential magnitudes. For astrometry, the separation error bar is $\pm 0.02^{\prime \prime}$ for $\rho \leq 1^{\prime \prime}, \pm 0.01^{\prime \prime}$ for $1^{\prime \prime}<\rho \leq 4^{\prime \prime}$, and $\pm 0.02^{\prime \prime}$ for $\rho>4^{\prime \prime}$. In Roberts et al. (2005) we mistakenly claimed that the position angle error is a constant. This is not true; the error in position angle caused by errors in determining the centroid of the secondary star location is bigger for systems with small separations than for those with larger separations. We have adopted $\pm 2^{\circ}$ for $\rho<1^{\prime \prime}$ and $\pm 1^{\circ}$ for $\rho>1^{\prime \prime}$ as our position angle error bar.

\section{RESULTS}

The rest of the paper is laid out in the following way: first, we discuss how we estimated a spectral type for any detected companions. Then we start discussing individual systems. We discuss observations of binaries for which we detected components that are already known in $\S 3.2$. Next, we discuss the detection of new components of binary systems in $\S 3.3$. The astrometry and photometry for all binaries is shown in Table 1 . Of course, we did not detect companions to all the stars we observed. These are described in Table 2. In $\S 3.5$, we discuss binaries for which we did not detect the previously known companions.

\subsection{Companion Spectral Type}

For stars where we detected another object in the field of view, we estimated the possible companion spectral type using the absolute magnitudes of the MK classification in Cox (2000). There are only data for luminosity classes V and I, so for luminosity classes IV and III we used the main-sequence data, while for luminosity classes II and I we used the supergiant data. We assumed that the companion was physically bound to the primary and was on the main sequence. If the primary is an unresolved binary, this will contaminate the spectral type of the primary and cause an error in the determination of the spectral type of the AO-resolved companion. The results are discussed in the sections detailing the individual stars.

Admittedly this is a fairly crude way of spectral typing, but it is all that is available until multifilter observations are done. With 
TABLE 2

Single B Stars

\begin{tabular}{|c|c|c|c|c|}
\hline HD & WDS & Hipparcos & Besselian Year & $\begin{array}{l}\text { FWHM } \\
(\operatorname{arcsec})\end{array}$ \\
\hline $224926 \ldots \ldots \ldots \ldots \ldots$ & $\ldots$ & 145 & 2002.6843 & 0.12 \\
\hline \multirow[t]{2}{*}{$225132 \ldots \ldots \ldots \ldots \ldots$} & $\ldots$ & 301 & 2002.6844 & 0.12 \\
\hline & & & 2002.6844 & 0.17 \\
\hline $225289 \ldots \ldots \ldots \ldots \ldots$ & $\ldots$ & 418 & 2002.6844 & 0.17 \\
\hline 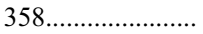 & $00084+2905$ & 677 & 2001.7423 & $0.12^{\mathrm{a}, \mathrm{b}}$ \\
\hline 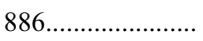 & $00132+1511$ & 1067 & 2001.7424 & $0.10^{\mathrm{b}}$ \\
\hline 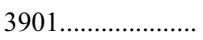 & $\ldots$ & 3300 & 2001.7425 & 0.14 \\
\hline $4142 \ldots \ldots \ldots \ldots \ldots \ldots$ & $\ldots$ & 3478 & 2002.6844 & 0.10 \\
\hline $4727 \ldots$ & & 3881 & 2001.7425 & 0.14 \\
\hline $10205 \ldots \ldots \ldots$ & $01406+4035$ & 7818 & 2001.7425 & $0.12^{\mathrm{b}}$ \\
\hline $12534 \ldots \ldots \ldots \ldots \ldots \ldots$ & $02039+4220$ & $\ldots$ & 2001.7426 & $0.12^{\mathrm{b}}$ \\
\hline 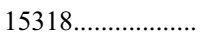 & $\ldots$ & 11484 & 2001.7426 & 0.12 \\
\hline $16582 \ldots \ldots \ldots \ldots \ldots$ & $\ldots$ & 12387 & 2001.7427 & 0.11 \\
\hline \multirow[t]{2}{*}{ 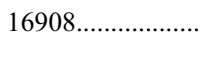 } & $\ldots$ & 12719 & 2001.7427 & 0.11 \\
\hline & & & 2001.7427 & 0.12 \\
\hline $17573 \ldots \ldots \ldots \ldots \ldots$ & $02500+2716$ & 13209 & 2001.7427 & $0.10^{\mathrm{c}}$ \\
\hline 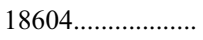 & $\ldots$ & 13954 & 2001.7428 & 0.12 \\
\hline \multirow[t]{2}{*}{$19356 \ldots \ldots \ldots \ldots \ldots$} & $03082+4057$ & 14576 & 2001.7456 & 0.08 \\
\hline & & & 2001.8656 & 0.10 \\
\hline $19698 \ldots \ldots \ldots \ldots \ldots \ldots$ & $\ldots$ & 14764 & 2001.7428 & 0.12 \\
\hline 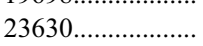 & $03475+2406$ & 17702 & 2001.7428 & $0.10^{\mathrm{b}}$ \\
\hline 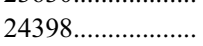 & $03541+3153$ & 18246 & 2001.7429 & $0.10^{\mathrm{b}}$ \\
\hline $24760 \ldots \ldots \ldots \ldots \ldots \ldots$ & $03579+4001$ & 18532 & 2001.7429 & $0.12^{\mathrm{b}}$ \\
\hline 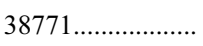 & $\ldots$ & 27366 & 2002.7392 & 0.23 \\
\hline $83754 \ldots \ldots \ldots \ldots \ldots \ldots$ & $\ldots$ & 47452 & 2002.2405 & 0.17 \\
\hline $86360 \ldots \ldots \ldots \ldots \ldots$ & $\ldots$ & 48883 & 2002.2405 & 0.10 \\
\hline 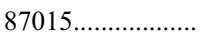 & $\ldots$ & 49220 & 2002.2406 & 0.08 \\
\hline 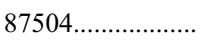 & $\ldots$ & 4940 & 2002.2406 & 0.09 \\
\hline \multirow[t]{2}{*}{$104337 \ldots \ldots \ldots \ldots \ldots \ldots$} & $\ldots$ & 58587 & 2002.2407 & 0.17 \\
\hline & & & 2002.2407 & 0.10 \\
\hline $106625 \ldots \ldots \ldots \ldots \ldots$ & $\ldots$ & 59803 & 2002.2408 & 0.26 \\
\hline $113797 \ldots \ldots \ldots \ldots \ldots$ & $\ldots$ & 63901 & 2002.2410 & 0.12 \\
\hline \multirow[t]{2}{*}{$116658 \ldots \ldots \ldots \ldots \ldots \ldots$} & $13252-1110$ & 65474 & 2002.2408 & $0.13^{\mathrm{b}}$ \\
\hline & & & 2002.2409 & $0.08^{\mathrm{b}}$ \\
\hline $120315 \ldots \ldots \ldots \ldots$ & $\ldots$ & 67301 & 2002.2410 & 0.08 \\
\hline $148688 \ldots \ldots \ldots \ldots \ldots$ & $\ldots$ & 80945 & 2001.3891 & 0.11 \\
\hline 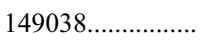 & $\ldots$ & 81122 & 2004.2589 & 0.23 \\
\hline $152235 \ldots \ldots \ldots \ldots \ldots$ & $\ldots$ & 82669 & 2001.3893 & 0.12 \\
\hline \multirow[t]{2}{*}{$154450 \ldots \ldots \ldots \ldots \ldots$} & $\ldots$ & $\ldots$ & 2001.4030 & 0.20 \\
\hline & & & 2001.4957 & 0.17 \\
\hline $158902 \ldots$ & $\cdots$ & 85881 & 2001.4686 & 0.12 \\
\hline $160762 \ldots$ & $17395+4600$ & 86414 & 2002.6839 & $0.16^{\mathrm{b}, \mathrm{c}}$ \\
\hline $163302 \ldots \ldots \ldots \ldots \ldots$ & $\ldots$ & $\ldots$ & 2001.3399 & 0.23 \\
\hline $164637 \ldots \ldots \ldots \ldots \ldots$ & $\ldots$ & 88396 & 2001.5179 & 0.12 \\
\hline $165516 \ldots \ldots \ldots \ldots \ldots . . . . . . .$. & $\ldots$ & 88760 & 2001.6711 & 0.11 \\
\hline $168987 \ldots \ldots \ldots \ldots . .$. & $\ldots$ & $\ldots$ & 2002.6785 & 0.14 \\
\hline \multirow[t]{2}{*}{$169454 \ldots \ldots \ldots \ldots . . . . .}$. & $\ldots$ & 90281 & 2001.6712 & 0.10 \\
\hline & & & 2001.7364 & 0.18 \\
\hline $170938 \ldots \ldots \ldots \ldots . . .$. & $\ldots$ & 90907 & 2001.6712 & 0.24 \\
\hline $171012 \ldots \ldots \ldots \ldots$ & $\ldots$ & 90950 & 2002.4518 & 0.12 \\
\hline 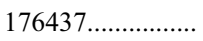 & $18589+3241$ & 93194 & 2002.6732 & $0.08^{\mathrm{b}}$ \\
\hline \multirow[t]{2}{*}{$177756 \ldots \ldots \ldots \ldots \ldots$} & $\ldots$ & 93805 & 2002.6839 & 0.12 \\
\hline & & & 2002.6841 & 0.12 \\
\hline $187235 \ldots \ldots \ldots \ldots \ldots$ & $19475+3824$ & 97376 & 2002.6843 & $0.14^{\mathrm{b}}$ \\
\hline $196867 \ldots \ldots \ldots \ldots \ldots$ & $20396+1555$ & 101958 & 2001.7420 & $0.10^{\mathrm{a}, \mathrm{b}}$ \\
\hline $207330 \ldots \ldots \ldots \ldots \ldots$ & $21468+4919$ & 107533 & 2001.7449 & $0.09^{\mathrm{a}}$ \\
\hline $212120 \ldots \ldots \ldots \ldots \ldots \ldots$ & $22210+4632$ & 110351 & 2001.7450 & $0.08^{\mathrm{b}}$ \\
\hline 213998.................. & $\ldots$ & 111497 & 2001.7449 & 0.09 \\
\hline
\end{tabular}

TABLE 2-Continued

\begin{tabular}{rcrcc}
\hline \hline HD & WDS & Hipparcos & Besselian Year & $\begin{array}{c}\text { FWHM } \\
(\operatorname{arcsec})\end{array}$ \\
\hline $214923 \ldots \ldots \ldots \ldots \ldots$ & $22415+1050$ & 112029 & 2001.7422 & $0.10^{\mathrm{b}}$ \\
$217675 \ldots \ldots \ldots \ldots \ldots$ & $23019+4220$ & 113726 & 2001.7423 & $0.12^{\mathrm{a}}$ \\
$217891 \ldots \ldots \ldots \ldots \ldots \ldots$ & $\ldots$ & 113889 & 2001.7449 & 0.08 \\
$218045 \ldots \ldots \ldots \ldots \ldots$ & $\ldots$ & 113963 & 2001.7423 & 0.12 \\
& & & 2001.7423 & 0.12 \\
\hline
\end{tabular}

${ }^{a}$ Last measured separation smaller than FWHM.

${ }^{b}$ Last measured separation too large for field of view.

${ }^{\mathrm{c}}$ Last measured separation should have been detected.

several filters, fairly accurate spectral typing can be done (ten Brummelaar et al. 2000).

The results from this analysis are shown in Table 3 . The table has the Henry Draper (HD) catalog number of the star, the $\Delta I$ from Table 1, the literature value for the spectral type of the primary, and the derived spectral type of the secondary. Stars for which the later part of the range was later than an M5 spectrum have the later type was listed as a dash.

\subsection{Known Binaries}

In this subsection of the paper, we discuss the measurements of known binary stars, for which we observed the previously known components. Section 3.3 discusses newly detected companions. The astrometry and photometry of all the binary stars are presented in Table 2. For each star we give the Washington Double Star (WDS) number, the discovery designation, the HD catalog number, the Hipparcos catalog number, the Besselian date of the observation, the separation in arcseconds, the position angle in degrees, and, finally, the differential magnitude measured in the Bessel $I$ band along with an error. We make notes of

TABLE 3

Companion Spectral Type

\begin{tabular}{|c|c|c|c|}
\hline $\mathrm{HD}$ & $\Delta I$ & SP1 & SP2 \\
\hline $3369 \ldots \ldots$ & $8.7 \pm 1.8$ & $\mathrm{~B} 5 \mathrm{~V}^{\mathrm{a}}$ & $\mathrm{K} 6 \mathrm{~V}-$ \\
\hline $5394 \ldots$ & $6.85 \pm 0.18$ & B0 IV ${ }^{b}$ & F2-G0 V \\
\hline $23862 .$. & $9.4 \pm 0.5$ & $\mathrm{~B} 8 \mathrm{nn}^{\mathrm{c}}$ & M5 V- \\
\hline $37711 \ldots .$. & $1.51 \pm 0.03$ & B3 $\mathrm{IV}^{\mathrm{d}}$ & B5-B8 V \\
\hline 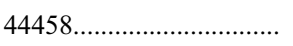 & $3.69 \pm 0.01$ & $\mathrm{~B} 1 \mathrm{~V}^{\mathrm{b}}$ & $\mathrm{B} 8-\mathrm{A} 0 \mathrm{~V}$ \\
\hline 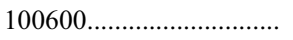 & $1.41 \pm 0.01$ & $\mathrm{~B} 4 \mathrm{~V}^{\mathrm{e}}$ & $\mathrm{B} 8-\mathrm{A} 0 \mathrm{~V}$ \\
\hline 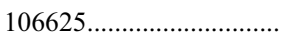 & $7.1 \pm 1.0$ & B8 III $^{\mathrm{f}}$ & K5-M5 V \\
\hline 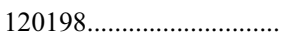 & $10.1 \pm 1.0$ & B9p $p^{g}$ & M8 V- \\
\hline 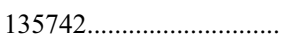 & $8.8 \pm 0.5$ & B8 $V^{h}$ & $\mathrm{M} 2 \mathrm{~V}-$ \\
\hline $157039 \ldots \ldots$ & $6.0 \pm 0.1$ & $\mathrm{~B} 4 \mathrm{Ia}^{\mathrm{i}}$ & $\mathrm{B} 5-\mathrm{A} 0 \mathrm{~V}$ \\
\hline 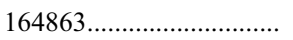 & $4.90 \pm 0.30$ & $\mathrm{~B} 0 \mathrm{~V}^{\mathrm{b}}$ & $\mathrm{A} 0-\mathrm{A} 5 \mathrm{~V}$ \\
\hline 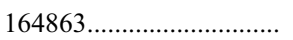 & $4.65 \pm 0.22$ & $\mathrm{~B} 0 \mathrm{~V}^{\mathrm{a}}$ & $\mathrm{A} 0-\mathrm{A} 5 \mathrm{~V}$ \\
\hline $164906 \ldots \ldots \ldots \ldots \ldots \ldots \ldots \ldots \ldots$ & $8.04 \pm 0.37$ & $\mathrm{~B} 1 \mathrm{IV}^{\mathrm{b}}$ & K2-M0 V \\
\hline 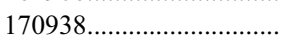 & $7.23 \pm 0.22$ & $\mathrm{~B} 1 \mathrm{Ia}^{\mathrm{b}}$ & $\mathrm{A} 0-\mathrm{A} 2 \mathrm{~V}$ \\
\hline $171432 .$. & $10.6 \pm 0.5$ & $\mathrm{~B} 1 \mathrm{Ia}^{\mathrm{b}}$ & $\mathrm{G} 2-\mathrm{K} 2 \mathrm{~V}$ \\
\hline 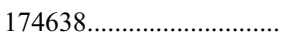 & $4.53 \pm 0.20$ & B8 $\mathrm{Iab}^{\mathrm{j}}$ & B2-B5 V \\
\hline 183914 & $4.12 \pm 0.17$ & B8 $V^{g}$ & $\mathrm{G} 2-\mathrm{G} 5 \mathrm{~V}$ \\
\hline
\end{tabular}

${ }^{\text {a }}$ Murphy (1969).

b Morgan et al. (1955).

${ }^{c}$ Cowley (1972).

${ }^{d}$ Edwards (1976).

${ }^{\mathrm{e}}$ Lesh (1968).

${ }^{\text {f }}$ Houk \& Smith-Moore (1988), p. 4.

g Cowley et al. (1969).

h Johnson \& Morgan (1953).

${ }^{i}$ Bidelman (1954).

${ }^{j}$ Hill et al. (1975). 
which systems are new discoveries and which ones are confirmation observations of prior binary detections.

In all but one case (see HD 37711), the observations of previously known stars agree with past observations. Coupled with our results from Roberts et al. (2005) this increases our confidence in the accuracy and reliability of our results.

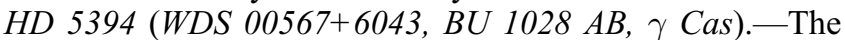
prototypical Be star, $\gamma$ Cas is in a multiple star system. Harmanec et al. (2000) detected a single-lined spectroscopic binary with a 203.59 day period. The spectroscopic companion's spectral type has not yet been determined. Gontcharov et al. (2000) found an astrometric binary with a period greater than $60 \mathrm{yr}$. The semimajor axis is greater than $0.3^{\prime \prime}$. We see no signs of this companion, but it could easily be a high dynamic range companion, which would be lost in the halo of the primary. Alternatively, the companion could be far from apastron and within the resolution limit of the image. This companion was also not seen in eight observations by Mason et al. (1997) and in a single measurement by Blazit et al. (1977). We do detect the long-known visual companion BU $1028 \mathrm{AB}$. We measure a differential magnitude in the $I$ band. The star is a known variable, with the $B$ and $V$ magnitudes fluctuating by several percent (Robinson et al. 2002). Presumably this variability extends to the $I$ filter. From the single measurement in the $I$ band, we estimate that the companion is an F2-G0 V star.

HD 37711 (WDS 05413+1632, BU 1007).- - This system has a grade 3 orbit $^{3}$ (Docobo \& Ling 1999). Astrometry computed from the orbital elements at the time of our observations, 2001.0985, gives a separation of $0.29^{\prime \prime}$ with a position angle of $244^{\circ}$. Our measurement of the separation, $0.26^{\prime \prime}$, agrees to within the errors, but the position angle is dramatically different, $221^{\circ}$. The star was observed before our observation on 1997.1255 (Hartkopf et al. 2000) and after our observation on 2004.095 (Scardia et al. 2005). Both measurements have a position angle of $242.8^{\circ}$. Obviously something is wrong with the measured position angle, although we are unable to pinpoint an exact cause. The problem most likely centers on the derotator in the Visible Imager system. While the control for it has been known to fail and produce anomalous results, no reports of errors exist for that time. It was also observed very early in the history of the system, at a time when the modes in the derotator were been reconfigured, and it is possible that the derotator was misprogrammed at that time. This object was the only star observed that night. This problem is not thought to extend to other stars in this paper.

The star was previously observed by our group with the $2.54 \mathrm{~m}$ Hooker telescope and its natural guide star AO system (ten Brummelaar et al. 2000) and AEOS (Roberts et al. 2005). The $\Delta I$ measurement from Mount Wilson on 1996.7902 was $1.52 \pm 0.18$, and the measurements from AEOS on 2002.9990 and 2003.0070 were $1.49 \pm 0.03$, while we get $1.51 \pm 0.03$ on 2001.0985 in this paper. Although this measurement was accidentally made in the $700-1060 \mathrm{~nm}$ filter, this filter has a very close transmission curve to the $I$ band. All the measurements are the same to within the errors. The system is listed in Baize \& Petit (1989) catalog of double stars with a variable component, but the variability is in doubt. The lack of variability seen in our five measurements with a time span of $6.2 \mathrm{yr}$ further increases this doubt.

Our one-filter determination of the companion spectral type agrees with the three-filter determination of ten Brummelaar et al. (2000). Ten Brummelaar et al. (2000) had a more specific subtype, but it shows that our one-filter determination is not totally unreasonable.

\footnotetext{
${ }^{3}$ See http://ad.usno.navy.mil/wds/orb6.html
}

HD 44458 (WDS 06214-1146, STF 3116 AB, FR CMa).Our astrometry measurements agree with the measurements listed in the WDS to within the scatter of the data points. Mason et al. (1997) did not resolve the companion, which was most likely due to the companion being too faint to be picked up by the CHARA speckle system. The primary is a Cepheid variable, so the differential magnitude fluctuates between measurements, making analysis harder. We determined that the secondary is a B8-A0 V star.

HD 100600 (WDS 11347+1648, STF $1552 \mathrm{AB})$.- - This system has been observed over 180 times, but the astrometry has not changed significantly. Our astrometry agrees with the previous measurements. From the differential magnitude, we determined that the secondary is a B8-A0 V star. The secondary is known to have variable radial velocity and is assumed to be a spectroscopic binary (Abt 1970), so this determination is most likely wrong and shows the weakness of determining spectral types from filter photometry.

HD 157039 (WDS 17227-3748, B908 AB).-The inner pair in this system has a single measurement of $\theta=113.7^{\circ}$ and $\rho=$ 2.66" from 1927.57 (Mason et al. 2001). That is very close to our measurement of $\theta=114^{\circ}$ and $\rho=2.71^{\prime \prime}$. We tried to determine if it was a physical pairing based on the Tycho-2 proper motions, but the error in the proper motion was too large to make a determination. From the differential magnitude, we determined that the companion is a $\mathrm{B} 5-\mathrm{A} 0 \mathrm{~V}$ star.

HD 164863 (WDS 18042-2230, B1975 BC).-The WDS lists four components to this system. The S698 AB pair has a separation of $29^{\prime \prime}$ and was last measured in 1999. The B1975 BC pair has a single measure of separation $2.02^{\prime \prime}$ and a position angle of $155.8^{\circ}$ in 1930.38 . The final pair is the ARA 1842 pair.

We measured the B1975 BC pair twice, on 2001.6710 and 2002.6729. Both observations show very similar astrometry, suggesting that the system is a slow mover. The Tycho- 2 proper motions and the measurements from the three epochs are inconsistent with the companion being a background star with no proper motion. It is likely that the system is physically bound and we are seeing orbital motion. Conclusive proof will require additional astrometry that can show nonlinear motion.

We determined that the companion is a $\mathrm{K} 2-\mathrm{M} 0 \mathrm{~V}$ star based on the differential magnitude. If the system is bound, it makes for a very interesting system with such a large mass ratio. Additional astrometric observations are warranted. The Tycho- 2 proper motions for the A component and the B component are not the same, strongly suggesting that the wide pair is nonphysical.

\subsection{New Discoveries \\ 3.3.1. Are These Real?}

With any search for new companions, especially faint ones, the question must be asked: Are these real detections, or is there another explanation? One possible explanation for false detections would be ghosts. The biggest argument against ghosts is that we do not see ghost companions in every data set. Also, the possible companions are not in the same orientation with respect to the primary star. We checked this by creating a map of the $(X, Y)$ positions of the pairs. We placed the primary at the center pixel of a square and the secondary at its relative separation and position angle. There was no discernible clumping to the locations of the secondaries. We created another image by combining all absolute $(X, Y)$ positions in a single image. There was no clumping of the secondary positions; as expected, the primary positions clumped slightly to the bottom left of the center. This is off from true center due to a small offset between the wave front sensor and the tip-tilt system. 

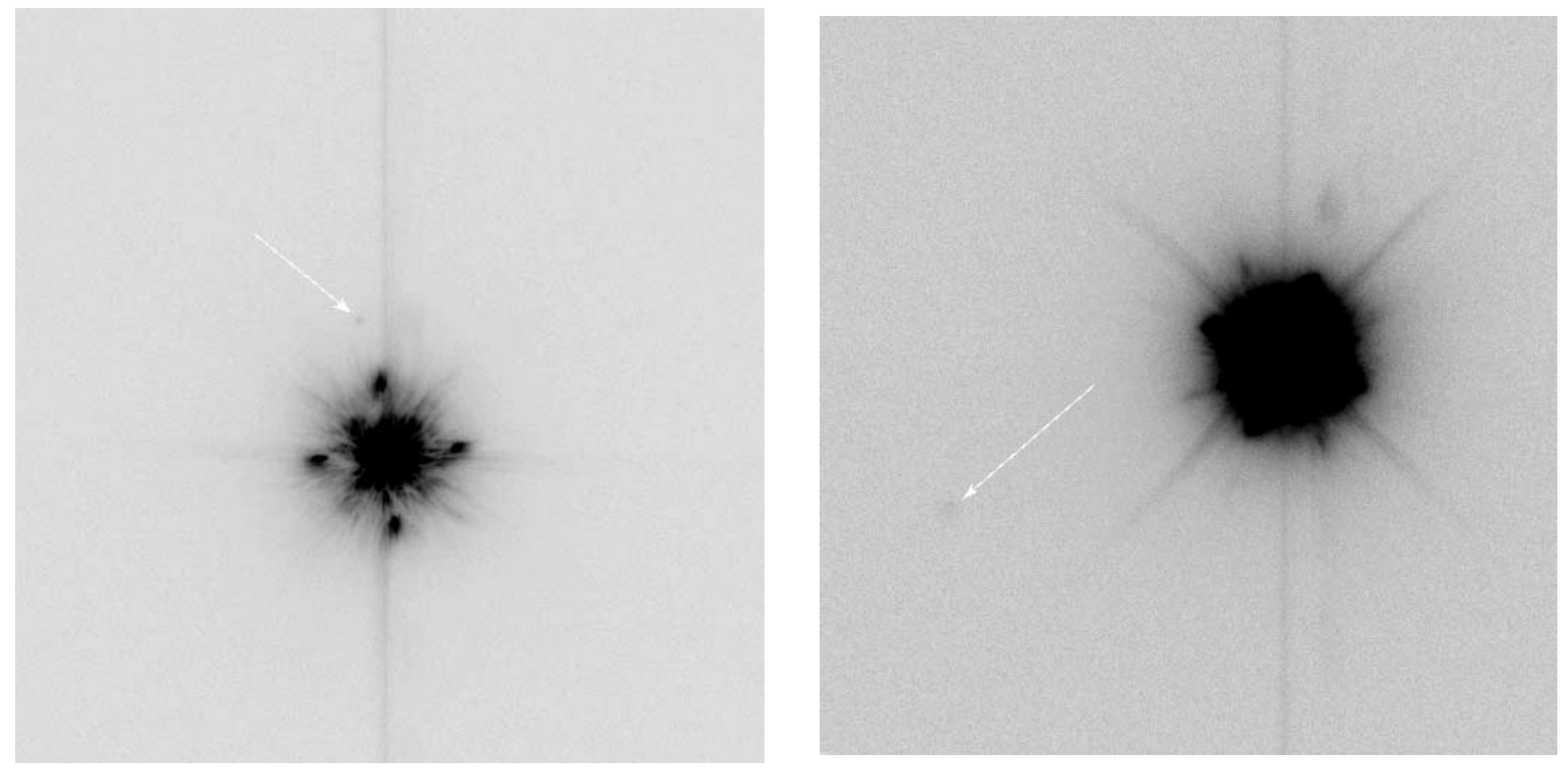

Fig. 1. - Two histogram-equalized and stretched $I$-band images of HD 19356 (Algol) taken 44 days apart. Both images show possible companions, but the companions have different astrometry. Arrows point to the location of the possible companions.

The optical path does change slightly for each star; one of three different neutral-density filters are used for the observations (since the science camera is only 12 bit, we often have to insert a neutraldensity filter to avoid saturation of the target star). We do not see any correlation between the new companions and the optical density filter used in the observation.

The most likely explanation for a false detection would be AO speckles (Racine et al. 1999). These speckles are found in the halo of the point-spread function (PSF) and are faint pointlike artifacts. These can arise from both the atmosphere and the optics of the telescope and AO system.

In analyzing data for this and other papers we have found possible companions that are not confirmed in subsequent observations. In some cases the subsequent image shows no companions, but at other times it shows a companion in a different spot. The exact cause of these false detections is not known. An example is shown in Figure 1. This shows two images of HD 19356 (Algol). On 2001.7456 we saw a companion at a separation of $1.91^{\prime \prime}$. Forty-four days later, on 2001.8656 , we saw a companion at a separation of 5.21". The image from day 272 is the sum of 1000 frames of data, and the image from day 316 is the sum of 250 frames. The change in separation is far too large to be explained by orbital motion or proper motion. In this paper, HD 19356 is the only known example of this behavior.

For observations where the subsequent observation fails to detect the companion, in addition to false detections, this could be because the companion is a variable and has faded in brightness, or, more likely, that the follow-up observation does not have as good a Strehl ratio as the first image, and the peak of PSF of the companion is below the detection threshold.

Roberts et al. (2005) detected a companion to HD 102510 (HIP 57562) with a $\Delta I$ of 7.9 ; this was confirmed by follow-up $J H K$ observations later that year. Turner et al. (2002) detected a companion to HD 161797 ( $\mu$ Her) with a dynamic range of $\Delta I$ of 7.26 and a $\Delta R$ of 9.29. This companion was confirmed by Debes et al. (2002). Metchev \& Hillenbrand (2004) detected a companion to HD 49197 with a $\Delta J$ of 9.6 and confirmed it at a later epoch. These examples show that it is possible to detect true companions at high dynamic ranges.

The hard part is determining which of the newly detected companions are real and which are not. Follow-up observations are key to answering this question, but unfortunately we were unable to make follow-up observations for any of the possible new companions discussed in this paper.

\subsubsection{Newly Discovered Companions}

In this subsection of the paper, we discuss the systems for which we have detected a possible new companion. We give each of these systems a WDS number based on the International Celestial Reference System coordinates and a discovery designation. Both of these have been communicated to the personnel maintaining the WDS catalog. For each system, we discuss the existing knowledge of the system, and we give the spectral type of the system determined from the $I$-band observation. The astrometry is given in Table 1 .

Three of the new discoveries have uncertain position angles: HD 3369, HD 23862, and HD 170938. There is a derotator in the AO system, but in addition to the standard mode it can also derotate the image so that the zenith vector is kept fixed; this simulates an instrument mounted on the side of the telescope. Prior to software upgrades in 2002, the position of the derotator was not reported in the data. The procedure was to take data during queue schedule observations in zenith-fixed mode to reduce confusion, but as we have no way of double-checking this, we felt it was better to report the uncertainty and both possible position angles. In Table 1 the position angle in zenith mode is listed with a "Z" and that in standard astronomical mode is listed with an "N." Careful reading of Table 1 shows several stars observed in 2001 with certain position angles. For stars with known position angles, we were able to determine the correct mode based on historical observations. HD 164906 was observed on the same night as HD 164863, a previously known binary; this allowed us to confirm that the zenith mode of the derotator was used.

HD 3369 (WDS 00369+3343, $\pi$ And ).- This a known multiple system; there are two wide visual companions, H5 17 Aa-B 
and H5 17 Aa-C. Shatskii (1998) determined that the Aa-C pair is a nonphysical optical binary. The central Aa pair is a double-line spectroscopic binary (Batten et al. 1978), and Hummell et al. (1995) measured the orbit with the Mark III interferometer. The system has been observed 15 times by multiple speckle-interferometry groups with no one detecting the companion we have detected, which is to be expected from the large dynamic range.

From the $I$-band differential magnitude, the companion would be later than a K6 V star. If physical, the separation is such that the system would be hierarchal and stable. It could also be a background star; additional measurements are needed either to measure the color or to measure orbital motion. As the possible new companion is quite close and has a high dynamic range, it could be a speckle and a false detection. However, the companion looks much like that in the observation of HD 23862 (see below), which is real.

HD 23862 ( $W D S 03492+2408)$. - The system primary is the famous star Pleione. It is a member of the Pleiades and has several known companions. The HL 28 Aa-B, HL 28 Aa-C, HL 28 Aa-D, and HL 28 Aa-E pairs all have separations over $1^{\prime}$. There is also the close CHR 125 Aa pair, which was discovered by McAlister et al. (1989). Mason et al. (1993) presented a few other measurements but also noted that it was undetected 8 times. It was also undetected by Balega et al. (1994). McAlister et al. (1989) said that the peaks of the autocorrelation are weak and indicative of a large dynamic range, which would only be detected under ideal seeing conditions. Pleione is a known variable star (Goraya et al. 1990), so the failures could be due to the fact that the dynamic range between the primary and companion changes and often exceeds the limit of speckle interferometry.

We do see the CHR 125 Aa pair, but fitstars is unable to converge on a solution because the AO PSF is quite odd due to poor performance of the AO system. Even though we cannot use fitstars, we can create an estimate of the astrometry. The estimated separation of the system is $0.24^{\prime \prime}$, which agrees with previous measurements. So we can say with confidence that we are detecting the companion and not a speckle. Follow-up observations are needed to measure the differential magnitude of the companion in several epochs to see if it is a fixed value or variable.

Pleione has also long been known to show radial velocity variations, and Luthardt \& Menchenkova (1994) determined that the system has a period of $35 \mathrm{yr}$ and concluded that this is the same as the CHR 125 Aa companion. Katahira et al. (1996) disagreed and found a period of 218 days, and concluded that the system is a triple system. Spectroscopic analysis is complicated by the shells of gas that the star throws out periodically. Additional observations with AO are needed, as it can help determine the orbit of CHR 125, which can be compared to the spectroscopic results.

We also detect a previously unknown companion with a separation of $4.66^{\prime \prime}$. The measured differential magnitude is $9.4 \pm$ 0.5 . Since the separation is over $4^{\prime \prime}$, it is probable that the image is suffering from anisoplanatism, in which the PSF of the primary and the secondary are slightly different. Since fitstars assumes that the PSFs are identical, this will cause an error in the results. We are not able to determine how much larger the errors are, but some caution should be exercised when using these results. The effects of anisoplanatism on AO binary star observations are discussed in Roberts et al. (2005). It is hard to determine at what separation anisoplanatism is a significant effect since it depends on the degree of AO correction and on the atmospheric turbulence at the time of the observations. As a rule of thumb we have chosen $4^{\prime \prime}$ as a break point based on experience.

If the system is physically bound, this would be a main-sequence $\mathrm{M}$ star or redder, which indicates that it is probably a background star. However, this determination is in considerable doubt because of the poor data quality, the variable nature of the primary, and the possible spectroscopic companion. These all corrupt the measurement of the differential magnitude. Further observations are needed to see whether this new system component is gravitationally bound.

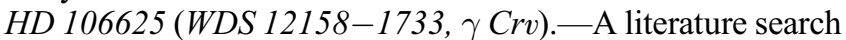
did not turn up any prior indications that this is a binary star. We have detected a companion with a separation of $1.06^{\prime \prime} \pm 0.01^{\prime \prime}$ and a $\Delta I$ of $7.1 \pm 1.0$. Based on the measured photometry, the companion would have a spectral type of K5-M5 V. Merrill (1922) did not resolve the binary with the Michelson interferometer on the $2.54 \mathrm{~m}$ Hooker telescope, nor did Brown et al. (1974) with the Narrabri Observatory intensity interferometer. Neither instrument would have detected a companion with the measured differential magnitude. Again, we are unable to determine if this is a background star or a physical companion.

HD 120198 (WDS 13466+5426, 84 UMa).- The system has a known wide companion, LDS 2914, with a separation of $70^{\prime \prime}$ (Mason et al. 2001). Abt \& Snowden (1973) found a constant radial velocity, indicating that the system is not a spectroscopic binary. The possible spectral type is later than an M8 V. This is a very late spectral type and suggests that the star is probably a background star.

HD 135742 (WDS 15170-0923, $\beta$ Lib).-We have detected a companion with a separation of $2.09^{\prime \prime} \pm 0.01^{\prime \prime}$ and a $\Delta I$ of $8.8 \pm 0.5$. McAlister et al. (1993) observed the system twice and did not resolve any companions, due to the high dynamic range. From the dynamic range, we estimate that the companion is later than a M2 V star. This increases the chance that this is a background star, but follow-up observations are needed.

HD 174638 (WDS 18501+3322, $\beta$ Lyr).-This is the prototype of the $\beta$ Lyr class of eclipsing binaries. It is composed of a pair of B stars, where one of the components is Roche lobe filling and donates mass to the other. The mass gainer is shrouded in an accretion disk, and there are numerous gas streams (Hubeny \& Plavec 1991). The WDS also lists five companions, all with separations greater than 45": STF 39 AB, BU 293 AC, BU 293 AD, BU $293 \mathrm{AE}$, and BU 293 AF. The B and F components are physical, while the others are not (Abt et al. 1962; Abt \& Levy 1976).

We have detected a possible companion with a separation of $0.54^{\prime \prime} \pm 0.02^{\prime \prime}$ and a differential magnitude of $4.53 \pm 0.20$. The differential magnitude suggests that the companion is a B2-B5 V star, but since the primary is actually the combination of two stars, one of which is shrouded in an accretion disk (Hubeny \& Plavec 1991), the determination of the new companion's spectral type is going to be difficult even with additional filters. It is possible that the companion is a speckle in the AO system masquerading as a star, although it is farther out from the primary and brighter than most speckles. If the companion is physical, it would form a hierarchal system.

Numerous observations with speckle interferometry have failed to show this companion (Bonneau et al. 1986; Miura et al. 1992, 1993, 1995; Isobe et al. 1992), but speckle interferometry would not be expected to see a companion with a $\Delta I$ of $4.53 \pm 0.20$. The system has also been extensively studied with spectroscopy. Harmanec \& Scholz (1993) reanalyzed 100 yr of radial velocity measurements. They detected possible slight variations of the systematic velocity of the system, which may arise from the blending effects of the circumstellar environment and do not require an unseen companion.

HD 164906 (WDS 18044-2423).-A literature search turned up no prior indications of this star being binary. We detected a possible companion with a separation of $3.14^{\prime \prime}$ and a $\Delta I$ of $8.04 \pm 0.37$. 
From the photometry, we estimate the companion's spectral type as $\mathrm{K} 2-\mathrm{M} 0 \mathrm{~V}$.

HD 170938 (WDS 18326-1542).-A literature search turned up no prior indications of this star being binary. We detected a possible companion with a separation of $7.31^{\prime \prime}$ and a $\Delta I$ of $7.23 \pm 0.22$. From the photometry, we estimate the companion's spectral type as A0-A2 V. The separation for this system is quite large and the image almost certainly suffers some degree of anisoplanatism. As with HD 23862, the error bars should be considered lower bounds on the true error.

HD 171432 (WDS 18356-1933).-A literature search turned up no prior indications of this star being binary. We detected a possible companion with a separation of $4.86^{\prime \prime}$ and a $\Delta I$ of $10.6 \pm$ 0.5 . From the photometry, we estimate the companion's spectral type as G2-K2 V. Again, the separation for this system is larger than $4^{\prime \prime}$, and the image probably suffers some degree of anisoplanatism. As with HD 23862 and HD 170938, the errors should be considered lower bounds on the true error.

HD 183914 (WDS 19307+2758, $\beta$ Cyg B, Alberio).-One of the most famous binary stars, Alberio has several components. The most widely known and best studied is the STF 43 Aa-B pair with its famous yellow and blue components, consisting of a K3 II and a B8 V star (Bidelman 1958). There is still uncertainty whether this pair is gravitationally bound (Griffin 1999).

There is a single measurement of a wider component, WAL 114 Aa-C, with a separation of 50", from 1944 (Mason et al. 2001). McAlister \& Hendry (1982) resolved the composite-spectra K3 II primary into a pair, MCA $55 \mathrm{Abc}$, with the new companion being later identified as a B0 V (ten Brummelaar et al. 2000). The MCA $55 \mathrm{Abc}$ pair is inconsistent with the pair found by Bonneau \& Foy (1980), BNU 10 Aab. This pair was later confirmed by Prieur et al. (2002). It is unlikely that they mistook the MCA 55 Abc pair for the BNU 10 Aab pair, since they also detected the MCA 55 Abc pair (Prieur et al. 2002). Several other attempts have failed to resolve this pair (Merrill 1922; Bonneau et al. 1980). The MCA $55 \mathrm{Abc}$ pair is physically bound and has had an orbit computed (Hartkopf 1999) albeit one with only partial phase coverage.

This presents a mystery. Why did the numerous speckle observations that resolved the MCA 55 Abc pair not detect the $\mathrm{BNU} 10 \mathrm{Aab}$ pair? One possibility is that the BNU $10 \mathrm{Aab}$ pair is a spurious detection; another possibility is that it is a star with a dynamic range that makes it difficult to detect. It would most likely be a background star, since if the BNU 10 Aab pair was physical it would not form a hierarchical system and would be unstable. If the background object was a variable star, this would add to difficulties, since sometimes it would be too faint to detect. Further observations are required with AO, although AEOS observations will most likely be unable to resolve the system due to the close separation.

We observed the B8 V companion and detected a companion with a separation of $0.39^{\prime \prime}$ and a $\Delta I$ of $4.12 \pm 0.17$. No companion was detected by Hartkopf \& McAlister (1984) or Mason et al. (1997), but the differential magnitude we measured would put the companion out of reach for the CHARA speckle system. At such a close separation and relatively small differential magnitude, the system is likely to be physical. Based on the spectral colors for main-sequence stars, the companion would be a G2-G5 V if it is a main-sequence star. Of course, a more exact and reliable determination would come from multiple-filter observations with AO.

\subsection{Single Stars}

Table 2 lists the stars for which no companions were detected. It lists the Hipparcos catalog number, the WDS number of the system if there is one, the HD catalog number, the observation date, and the full width at half-maximum (FWHM) of the observed PSF. The FWHM provides a useful gauge for estimating the minimum separation resolvable by the observations and also provides a metric of AO performance, which varies from night to night as a function of atmospheric conditions, target brightness, and air mass.

\subsection{Unresolved Binary Stars}

Some of the stars listed in Table 2 are part of multiple star systems listed in the WDS. The AEOS AO science camera has a field of view of $10^{\prime \prime}$, and normally the target star is placed near center of the image. Because of this, we are unable to detect companions with separations greater than $\sim 5^{\prime \prime}$ (the central star is not always placed in the exact center of the field of view). Many of the known binaries have separations greater than this, and we have marked those systems in Table 2. In this section, we comment on the systems that we had some chance of detecting.

HD 358 (WDS 00084+2905).- - This object has three known components. The MKT 11 Aa orbit computed by Pourbaix (2000) predicts a separation of $0.02^{\prime \prime}$ at the time of observation, which is too close to be resolved by AEOS AO. The wider pair is too wide to be detected with our field of view.

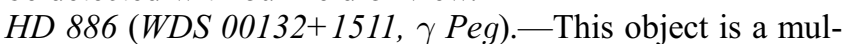
tiple star system with two known visual companions, both of which are listed in the WDS as having separations of approximately $3 \prime$. Chapellier et al. (2006) detected a spectroscopic companion in a 370.5 day orbit. They also detected a change in the period, which in other stars has been explained as the interaction with a companion in period with an orbit lasting tens of years. McAlister et al. (1989) did not detect any companions with speckle interferometry, and our search has also failed to turn up anything. Without knowing where the companion is, we cannot provide a definitive upper bound for its brightness, since the detectable dynamic range is a function of separation. However, the dynamic range figure in N. Turner et al. (2007, in preparation) provides a useful guide.

HD 17573 (WDS 02500+2716, 41 Ari).-This object has at least five components. We only had a chance of resolving the MCA 10 Aa pair; the rest are too wide to be detected. This system is a spectroscopic binary, and the WDS lists it as being resolved 4 times, with three negative detections. The astrometry measured closest to our observation was $\rho=0.118^{\prime \prime}$ and $\theta=180.1^{\circ}$ in 2003.9518 (W. Hartkopf et al. 2007, in preparation). On 2001.7427, we did not see any sign of a companion, and the measured FWHM of the PSF was $0.10^{\prime \prime}$. This would make it difficult to detect a companion with nearly the same separation. There was no significant broadening of the PSF, which would indicate a barely resolved companion.

HD 160762 (WDS 17395+4600, ८ Her).-This object has three known visual components. The BU 1459 Aa-B pair is much too wide to be detected in our observations, but the inner BLA 5 Aa pair has a single measurement with a $\rho$ of $0.165^{\prime \prime}$ and a $\theta$ of $6^{\circ}$ from 1975 (Blazit et al. 1977). The FWHM of our observation is $0.16^{\prime \prime}$, and it is likely that this companion has moved within this limit on our observation. Kodaira (1971) and Abt \& Levy (1978) determined that the system also contained a spectroscopic binary with a period of $\sim 113$ days. The primary is a slowly pulsating B star, which makes analysis of the radial velocities complicated, but Chapellier et al. (2000) were able to compute the period as $112.825 \pm 0.0008$ days and the mass fraction as $0.0013 \pm 0.0004 M_{\odot}$.

HD 196867 (WDS 20396+1555).-While this object has multiple components, all but the WCK 2 Aa pair are much too wide to be detected by the AEOS system. The orbital elements 
from Soderhjelm (1999) predict that WCK 2 Aa would have a separation of $0.095^{\prime \prime}$ during the time of our observation, which is just within the measured FWHM of the AO image.

HD 207330 (WDS 21468+4919, MIU 3).- -This object has only a single resolved pair. The Fourth Interferometric Catalog 4 lists measurements from 1990 to 1996 with the pair being intermittently resolved and then unresolved. We also failed to resolve the system, even though the PSF had a FWHM of $0.09^{\prime \prime}$. The primary is also a spectroscopic binary with an orbital period of 72.0162 days, but the orbit is considered preliminary (Batten et al. 1978).

\section{SUMMARY}

We have carried out a small survey of B stars for companions with the AEOS AO system in the $I$ band. We have detected the previously known companions that had observable separations. We have detected 10 possible new companions. Of these, four (HD 3369, HD 23862, HD 120198, and HD 135742) are probably not physically bound since our single-filter spectral-type determination suggests that the star is cooler than an M5 V star. While it

\footnotetext{
${ }^{4}$ The Fourth Interferometric Catalog can be found at http://ad.usno.navy.mil/ wds/int 4 .html.
}

is possible that these companions are white or brown dwarfs, it is much more likely that the star is a background star. All of these 10 companions need follow-up observations in multiple filters to confirm their existence and determine the spectral type. Determining the spectral type is key to understanding whether they are gravitationally bound to the B star or merely background objects. This is key to understanding the true binary fraction of B stars.

We thank Brian Mason and Bill Hartkopf for providing data from the WDS Catalog and useful and insightful comments. Also, we thank the numerous staff members of the Maui Space Surveillance System who helped make these data possible. The US Air Force provided the telescope time, on-site support, and $80 \%$ of the research funds for this AFOSR and NSF jointly sponsored research under grant number NSF AST 00-88498. L. C. R. was funded by AFRL/DE contract number F29601-00-D-0204. T. A. t. B. was supported by the Center for High Angular Resolution Astronomy at Georgia State University. This research made use of the Washington Double Star Catalog, maintained at the US Naval Observatory, the SIMBAD database, operated by the CDS in Strasbourg, France, and NASA's Astrophysics Data System.
Abt, H. A. 1970, ApJS, 19, 387

Abt, H. A., Gomez, A. E., \& Levy, S. G. 1990, ApJS, 74, 551

Abt, H. A., Jeffers, H. M., Gibson, J., \& Sandage, A. R. 1962, ApJ, 135, 429

Abt, H. A., \& Levy, S. G. 1976, AJ, 81, 659 1978, ApJS, 36, 241

Abt, H. A., \& Snowden, M. S. 1973, ApJS, 25, 137

Baize, P., \& Petit, M. 1989, A\&AS, 77, 497

Balega, I. I., et al. 1994, A\&AS, 105, 503

Batten, A. H., Fletcher, J. M., \& Mann, P. J. 1978, Publ. Dominion Astrophys.

Obs. Victoria, 15,121

Bidelman, W. P. 1954, PASP, 66, 249 1958, PASP, 70, 168

Blazit, A., Bonneau, D., Koechlin, L., \& Labeyrie, A. 1977, ApJ, 214, L79

Bonneau, D., Balega, Y., Blazit, A., Foy, R., Vakili, F., \& Vidal, J. L. 1986, A\&AS, 65, 27

Bonneau, D., Blazit, A., Foy, R., \& Labeyrie, A. 1980, A\&AS, 42, 185

Bonneau, D., \& Foy, R. 1980, A\&A, 86, 295

Bradley, E. S., Roberts, L. C., Jr., Bradford, L. W., Skinner, M. A., Nahrstedt, D. A., Waterson, M. F., \& Kuhn, J. R. 2006, PASP, 118, 172

Brown, H. R., Davis, J., \& Allen, L. R. 1974, MNRAS, 167, 121

Chapellier, E., Le Contel, D., Le Contel, J. M., Mathias, P., \& Valtier, J.-C. 2006, A\&A, 448, 697

Chapellier, E., Mathias, P., Le Contel, J.-M., Garrido, R., Le Contel, D., \& Valtier, J.-C. 2000, A\&A, 362, 189

Cowley, A. 1972, AJ, 77, 750

Cowley, A., Cowley, C., Jaschek, M., \& Jaschek, C. 1969, AJ, 74, 375

Cox, A. N. 2000, Allen's Astrophysical Quantities (4th ed.; New York: Springer)

Debes, J. H., Ge, J., \& Chakraborty, A. 2002, ApJ, 572, L165

Docobo, J. A., \& Ling, J. F. 1999, ApJS, 120, 41

Edwards, T. W. 1976, AJ, 81, 245

Gontcharov, G. A., Andronova, A. A., \& Titov, O. A. 2000, A\&A, 355, 1164

Goraya, P. S., Tur, N. S., Sharma, S. D., \& Malhi, J. S. 1990, Ap\&SS, 174, 1

Griffin, R. F. 1999, J. R. Astron. Soc. Canada, 93, 208

Harmanec, P., \& Scholz, G. 1993, A\&A, 279, 131

Harmanec, P., et al. 2000, A\&A, 364, L85

Hartkopf, W. I. 1999, IAUDS, Inf. Circ. 139

Hartkopf, W. I., \& McAlister, H. A. 1984, PASP, 96, 105

Hartkopf, W. I., et al. 2000, AJ, 119, 3084

Hill, G., Hilditch, R. W., Younger, F., \& Fisher, W. A. 1975, MmRAS, 79, 131

Houk, N., \& Smith-Moore, M. 1988, Michigan Spectral Survey (Ann Arbor: Univ. Michigan)

Hubeny, I., \& Plavec, M. J. 1991, AJ, 102, 1156

Hummell, C. A., Armstrong, J. T., Buscher, D. F., Mozurkewich, D., Quirrenback, A., \& Vivekanand, M. 1995, AJ, 110, 376

Isobe, S., Noguchi, M., Ohtsubo, J., Baba, N., Miura, N., Tanaka, T., \& Ni-Ino, M. 1992, Publ. Natl. Astron. Obs. Japan, 2, 459

\section{REFERENCES}

Johnson, H. L., \& Morgan, W. W. 1953, ApJ, 117, 313

Katahira, J., Hirata, R., Ito, M., Katoh, M., Ballereau, D., \& Chauville, J. 1996, PASJ, 48, 317

Kodaira, K. 1971, PASJ, 23, 159

Lesh, J. R. 1968, ApJS, 17, 371

Luthardt, R., \& Menchenkova, E. V. 1994, A\&A, 284, 118

Mason, B. D. 1994, Ph.D. thesis, Georgia State Univ.

Mason, B. D., Hartkopf, W. I., McAlister, H. A., \& Sowell, J. R. 1993, AJ, 106, 637

Mason, B. D., ten Brummelaar, T. A., Gies, D. R., Hartkopf, W. I., \& Thaller, M. L. 1997, AJ, 114, 2112

Mason, B. D., Wycoff, G. L., Hartkopf, W. I., Douglass, G. G., \& Worley, C. E. 2001, AJ, 122, 3466

McAlister, H. A., Hartkopf, W. I., Hutter, D. J., \& Franz, O. G. 1987, AJ, 93, 688

McAlister, H. A., Hartkopf, W. I., Sowell, W. R., Dombrowski, E. G., \& Franz, O. G. 1989, AJ, 97, 510

McAlister, H. A., \& Hendry, E. M. 1982, ApJS, 48, 273

McAlister, H. A., Mason, B. D., Hartkopf, W. I., \& Shara, M. M. 1993, AJ, 106,1639

Merrill, P. W. 1922, ApJ, 56, 40

Metchev, S. A., \& Hillenbrand, L. A. 2004, ApJ, 617, 1330

Miura, N., Baba, N., Ni-Ino, M., Ohtsubo, J., Noguchi, M., \& Isobe, S. 1992, Publ. Natl. Astron. Obs. Japan, 2, 561

Miura, N., Iribe, T., \& Kubo, T. 1995, Publ. Natl. Astron. Obs. Japan, 4, 67

Miura, N., Ni-Ino, M., Baba, N., Iribe, T., \& Isobe, S. 1993, Publ. Natl. Astron. Obs. Japan, 3, 153

Morgan, W. W., Code, A. D., \& Whitford, A. E. 1955, ApJS, 2, 41

Murphy, R. E. 1969, AJ, 74, 1082

Pourbaix, D. 2000, A\&AS, 145, 215

Prieur, J.-L., et al. 2002, ApJS, 142, 95

Racine, R., Walker, G. A. H., Nadeau, D., Doyon, R., \& Marois, C. 1999, PASP, 111, 587

Roberts, L. C., Jr., \& Neyman, C. R. 2002, PASP, 114, 1260

Roberts, L. C., Jr., et al. 2005, AJ, 130, 2262

Robinson, R. D., Smith, M. A., \& Henry, G. W. 2002, ApJ, 575, 435

Scardia, M., Prieur, J.-L., Sala, M., Ghigo, M., Koechlin, L., Aristidi, E., \& Mazzoleni, F. 2005, MNRAS, 357, 1255

Shatskii, N. I. 1998, Pis'ma Astron. Zh., 24, 307

Soderhjelm, S. 1999, A\&A, 341, 121

ten Brummelaar, T. A., Mason, B. D., Bagnuolo, W. G., Jr., Hartkopf, W. I., McAlister, H. A., \& Turner, N. H. 1996, AJ, 112, 1180

ten Brummelaar, T. A., Mason, B. D., McAlister, H. A., Roberts, L. C., Jr., Turner, N. H., Hartkopf, W. I., \& Bagnuolo, W. G., Jr. 2000, AJ, 119, 2403 Turner, N. H., ten Brummelaar, T. A., McAlister, H. A., Mason, B. D., Hartkopf, W. I., \& Roberts, L. C., Jr. 2001, AJ, 121, 3254

Wolff, S. C. 1978, ApJ, 222, 556 